\title{
Interventional 4-D Motion Estimation and Reconstruction of Cardiac Vasculature without Motion Periodicity Assumption ${ }^{\star}$
}

\author{
Christopher Rohkohl ${ }^{1,2}$, Günter Lauritsch ${ }^{2}$, Marcus Prümmer ${ }^{1}$, \\ and Joachim Hornegger ${ }^{1}$ \\ 1 Chair of Pattern Recognition, Department of Computer Science,
} Friedrich-Alexander University Erlangen-Nuremberg, 91058 Erlangen, Germany

${ }^{2}$ Siemens AG, Healthcare Sector, 91301 Forchheim, Germany

\begin{abstract}
Anatomical and functional information of cardiac vasculature is a key component of future developments in the field of interventional cardiology. With the technology of C-arm CT it is possible to reconstruct intraprocedural 3-D images from angiographic projection data. Current approaches attempt to add the temporal dimension (4D) by ECG-gating in order to distinct physical states of the heart. This model assumes that the heart motion is periodic. However, frequently arrhytmic heart signals are observed in a clinical environment. In addition breathing motion can still occur. We present a reconstruction method based on a 4-D time-continuous motion field which is parameterized by the acquisition time and not the quasi-periodic heart phase. The output of our method is twofold. It provides a motion compensated 3-D reconstruction (anatomic information) and a motion field (functional information). In a physical phantom experiment a vessel of size $3.08 \mathrm{~mm}$ undergoing a non-periodic motion was reconstructed. The resulting diameters were $3.42 \mathrm{~mm}$ and $1.85 \mathrm{~mm}$ assuming non-periodic and periodic motion, respectively. Further, for two clinical cases (coronary arteries and coronary sinus) it is demonstrated that the presented algorithm outperforms periodic approaches and is able to handle realistic irregular heart motion.
\end{abstract}

\section{Introduction}

One key component of future developments in the field of interventional cardiology is three-dimensional image information before, during and after interventional procedures, e.g. pacemaker implantations or ablation procedures. With the technology of C-arm CT it is possible to reconstruct intraprocedural 3-D images from angiographic projection data [1]. However, cardiac reconstruction is yet a challenging problem due to the long acquisition time of several seconds at which a couple of heart beats occur, leading to motion related image artifacts, e.g. blurring or streaks.

\footnotetext{
* We thank Prof. Dr. med. J. Brachmann, Dr. med. K. Gutleben and Dr. med. H. Rittger from the Klinikum Coburg, Germany for providing clinical image data.
} 
An established technique for time-resolved cardiac reconstruction is to record the electrocardiogram (ECG) during the data acquisition. Based on the ECGsignal a relative cardiac phase is assigned to each projection image assuming a cyclic heart motion [2]. The phase information is used for a phase-correlated reconstruction by gating or motion estimation and compensation. A gated reconstruction takes only those images into account that lie inside a defined temporal window, that is centered at the targeting reconstruction phase [3,4. This is however not ideal in terms of missing data and residual motion. To increase the data usage motion compensated reconstruction algorithms are applied. The phase information is used during motion estimation to parameterize a motion field that maps every heart phase to the target phase by some kind of registration operation [5, 6, 7, 8]. The common problem of such approaches is that the averaged periodic motion model does not necessarily represent accurately the actual heart motion of each individual beat. Thus the quality of the motion correction and periodicity assumption are correlated.

Accordingly, the previous methods were shown to provide reasonable results in the presence of regular heart rates without breathing or other patient motion. However, in the field of intraprocedural cardiac reconstruction, the patients suffer from heart diseases and cannot completely hold breath, stay still or have irregular heart beats. Those aspects do conflict with the periodicity assumption. Up to now, these problems were addressed by approximate $2-\mathrm{D}$ corrections in the projection image. Blondel et al. 5] proposed to model breathing motion of the heart as a translation mainly in axial direction. Hansis et al. 9] proposed to cope with the problem by performing a 2-D/2-D registration of the projection image with a forward projection of an initial reconstruction. However, none of the methods can cope with the general case of non-cyclic 3-D motion. In this paper a method for the 4-D reconstruction of selectively contrasted cardiac vasculature without perodicity assumption is introduced. This is achieved by two major contributions: First, the introduction of a time-continous motion field which is parameterized by the acquisition time and not the periodic heart phase. Second, the development of a theoretically founded, fast and robust motion estimation algorithm which is based on an extension of a FDK reconstruction to dynamic objects and thus couples motion estimation and reconstruction.

\section{Methods}

The presented motion estimation scheme is built up from two components. First, an initial reference reconstruction is performed using an ECG-gated or even nongated reconstruction. This reference image is post-processed such that only the vasculature is visible. Second, the parameters of the motion model are estimated by optimizing an objective function. The objective function aims to maximize the joint intensity of the reference image and the motion compensated FDK reconstruction.

This part of the paper is organized to contain a step-wise development of the required methods. In Sect. 2.1 the parametric motion model is presented. 
Next, the motion model is introduced into the FDK reconstruction in Sect. 2.2. Prepared with both concepts, the objective function for motion estimation is subject of Sect. 2.3. Finally, in Sect. 2.4 the optimization strategy is discussed.

\subsection{4-D B-Spline Motion Model}

We assume a time-continous motion model that maps a voxel $\boldsymbol{x}=\left(x_{0}, x_{1}, x_{2}\right)^{T}$ to a new voxel location $\boldsymbol{x}^{\prime}$ for each projection image. It is conceptionalized by a function $M: \mathbb{N} \times \mathbb{R}^{3} \times \mathrm{P} \mapsto \mathbb{R}^{3}$ with $M(i, \boldsymbol{x}, \boldsymbol{r})=\boldsymbol{x}^{\prime}$ transforming the voxel coordinate $\boldsymbol{x}$ at the time of the $i$-th projection image. The mapping is based on the motion model paramters $\boldsymbol{r} \in \mathrm{P}$. In this work, a 4-D B-spline is used. A set of $C_{j} \times C_{k} \times C_{l} \times C_{t}$ control points is placed uniformly in space and time. Each control point is assigned a displacement vector, forming the set of motion model parameters $\mathrm{P}=\left\{\boldsymbol{r}_{j k l t} \in \mathbb{R}^{3} \mid 1 \leq j, k, l, t \leq C_{j}, C_{k}, C_{l}, C_{t}\right\}$. Formally, the motion model is then given by

$$
M(i, \boldsymbol{x}, \boldsymbol{r})=\boldsymbol{x}+\sum_{j, k, l, t} B_{j}\left(x_{0}\right) B_{k}\left(x_{1}\right) B_{l}\left(x_{2}\right) B_{t}(i) \boldsymbol{r}_{j k l t} \quad \forall j, k, l, t,
$$

with $\{B$.$\} being the set of cubic B-spline basis functions [10].$

\subsection{Dynamic FDK-Reconstruction}

The formulation of our objective function for motion estimation (Sect. 2.3) is based on a dynamic reconstruction algorithm $f(\boldsymbol{x}, \boldsymbol{r})$. The function $f$ returns the reconstructed object value at a voxel $\boldsymbol{x}$ based on the motion model parameters $\boldsymbol{r}$. In principle, any dynamic reconstruction algorithm could be used. In this paper, an extension of the FDK reconstruction method for moving objets is utilized [3, 7 .

Formally, the dynamic FDK reconstruction $f: \mathbb{R}^{3} \times \mathrm{P} \mapsto \mathbb{R}$ is given by

$$
f(\boldsymbol{x}, \boldsymbol{r})=\sum_{i} \underbrace{w(i, M(i, \boldsymbol{x}, \boldsymbol{r}))}_{=s_{1}} \cdot \underbrace{p(i, A(i, M(i, \boldsymbol{x}, \boldsymbol{r})))}_{=s_{2}} .
$$

The function $w: \mathbb{N} \times \mathbb{R}^{3} \mapsto \mathbb{R}$ is the distance weight of the FDK-formula. The pre-processed, filtered and redundancy weighted projection data is accessed by the function $p: \mathbb{N} \times \mathbb{R}^{2} \mapsto \mathbb{R}$ where $p(i, \boldsymbol{m})$ returns the value of the $i$-th projection image at the pixel $\boldsymbol{m}$. The perspective projection is given by the function $A: \mathbb{N} \times \mathbb{R}^{3} \mapsto \mathbb{R}^{2}$, where $A(i, \boldsymbol{x})=\boldsymbol{m}$ maps a voxel $\boldsymbol{x}$ to a pixel location $\boldsymbol{m}$ in the $i$-th projection image. The shortcuts $s_{1}, s_{2}$ are introduced for convenience and are reused in Sect. 2.4.

\subsection{Objective Function for Motion Estimation}

The proposed approach for motion estimation is inspired by the works of Rohkohl et al. [7] and Hansis et al. 9]. Their results suggest that a sufficient initial reconstruction can be obtained by a FDK-like ECG-gated reconstruction which 
can be improved by motion estimation and compensation [7,9]. Our method also requires such an inital reconstruction, which is post-processed such that only the sparse and contrasted vascular tree is visible. It is obtained by applying a transfer function in a volume renderer [7]. In the following the resulting reference image is denoted $f_{r}$. Regarding the quality of $f_{r}$ it is especially important that larger structures are present. Due to the fact that the motion field is smooth in space and time, smaller structures are likely to move in accordance with the larger structures. Thus they will become visible in a motion corrected reconstruction.

Formally, motion estimation is formulated as a multi-dimensional optimization problem where the motion model parameters $\hat{\boldsymbol{r}} \in \mathrm{P}$ minimizing the objective function $\mathcal{O}: \mathrm{P} \mapsto \mathbb{R}$ need to be estimated, i.e.

$$
\hat{\boldsymbol{r}}=\arg \min _{\boldsymbol{r} \in \mathrm{P}} \mathcal{O}(\boldsymbol{r})=\arg \min _{\boldsymbol{r} \in \mathrm{P}}(\mathcal{L}(\boldsymbol{r})+\mathcal{R}(\boldsymbol{r})),
$$

with $\mathcal{R}$ being a regularizer and $\mathcal{L}$ being a dissimilarity measure. We decided for the dissimilarity function proposed by Rohkohl et al. 7. It aims to maximize the joint intensity of the reference image $f_{r}$ and another reconstruction - in our case the dynamic reconstruction $f(\boldsymbol{x}, \boldsymbol{r})$. It is given by

$$
\mathcal{L}(\boldsymbol{r})=-\sum_{\boldsymbol{x}} f_{r}(\boldsymbol{x}) \cdot f(\boldsymbol{x}, \boldsymbol{r})
$$

During the estimation of the motion model parameters, it needs to be ensured that only plausible motions are considered, i.e. no rapid motion or folding. Numerous regularizers have already been proposed for B-spline based motion models, e.g. [5,7] and hence will not be covered in this paper.

\subsection{Optimization Strategy and Runtime Considerations}

Many advanced optimization strategies require the gradient of (3), e.g. the LBFGS-B algorithm [11]. The derivative of (3) with respect to the motion model parameters is given by

$$
\frac{\partial \mathcal{O}(\boldsymbol{r})}{\partial \boldsymbol{r}}=\frac{\partial \mathcal{L}(\boldsymbol{r})}{\partial \boldsymbol{r}}+\frac{\partial \mathcal{R}(\boldsymbol{r})}{\partial \boldsymbol{r}}=-\sum_{\boldsymbol{x}}\left(f_{r}(\boldsymbol{x}) \frac{\partial f(\boldsymbol{x}, \boldsymbol{r})}{\partial \boldsymbol{r}}\right)+\frac{\partial \mathcal{R}(\boldsymbol{r})}{\partial \boldsymbol{r}} .
$$

It contains the derivative $\frac{\partial f}{\partial r}$ of the dynamic reconstruction (2) with respect to the motion model parameters. The derivative can be calculated analytically and is given by

$$
\frac{\partial f(\boldsymbol{x}, \boldsymbol{r})}{\partial \boldsymbol{r}}=\sum_{i}\left(\frac{\partial w}{\partial M} s_{2}+s_{1} \frac{\partial p}{\partial A} \frac{\partial A}{\partial M}\right) \frac{\partial M(i, \boldsymbol{x}, \boldsymbol{r})}{\partial \boldsymbol{r}} .
$$

The computation of the objective function and the corresponding derivative is fast as only non-zero voxel of the reference image $f_{r}$ contribute to the objective function value. In most cases the number of non-zero voxels is $<1 \%$. This property can be exploited for obtaining a fast implementation. Further, the dynamic FDK reconstruction is highly parallelizable and can be efficiently implemented on modern hardware, e.g. the GPU [12]. 


\section{Evaluation}

\subsection{Methods and Materials}

Evaluation Strategy. In order to assess the performance of the proposed algorithm, the reconstruction results of our method are compared to the approach of periodic motion [7]. In the periodic approach, ECG-gated reconstructions of several heart phases are registered with the reference image $f_{r}$ by maximizing the joint intensity. For the presented results, ten disjoint ECG-gated subreconstructions have been performed using each $10 \%$ of all projection data. Both algorithms are provided with equivalent parameter settings. As optimizer, the L-BFGS-B algorithm [11] with a fixed number of 50 iterations was used. All datasets were acquired on an Artis zee C-arm system (Siemens AG, Healthcare Sector, Germany) covering an angular range of $200^{\circ}$.

Physical Heart Phantom. A physical heart phantom has been built from an elastic material filled with water. At a rate of $50 \mathrm{bpm}$ the water is pumped in and out causing an elastic motion. In addition, the phantom is mounted on a compensator for a slow breathing motion of approx. 0.5 cycles per second. The artificial heart is surrounded by tubular structures filled with contrast agent mimicing the vascular system. An ECG-like signal is generated for the cardiac motion.

In total, three different datasets have been acquired. Each dataset consists of $N=133$ projection images with an acquisition time of $T=5$ seconds. The first dataset is the static phantom. It is used to determine the ground truth reconstruction. The second dataset is the moving phantom undergoing periodic cardiac motion. It is used to test the algorithms on cyclic motion patterns. The third dataset is the moving phantom undergoing a non-periodic combination of cardiac and respiratory motion. It is used to assess the algorithm performance on non-cyclic motion patterns.

For determing the quality of the reconstructions, a visual inspection of the volume renderings of all datasets using the same window settings, is performed. In addition, the vessel diameter, determined from MPR-views, is compared at a representative vessel position.

Clinical Cases: Arterial and Venous Vascular System. In order to demonstrate the clinical feasibility of the presented concepts, the algorithm is applied to the reconstruction of the venous and arterial vascular system. The first case is a coronary artery dataset with $N=133$ and $T=5$ seconds. The second case is a coronary sinus dataset with an $N=397$ and $T=8$ seconds. For the latter case, no ECG-signal is available due to a mislocation of the electrode. Periodic approaches cannot handle this situation. Our approach is feasible when using the non-gated reconstruction as reference image $f_{r}$. 


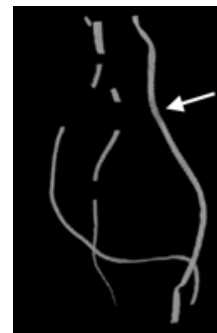

(a)

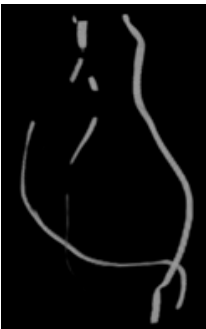

(b)

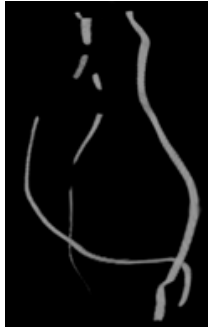

(c)

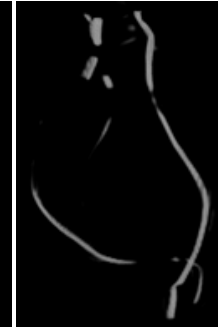

(d)

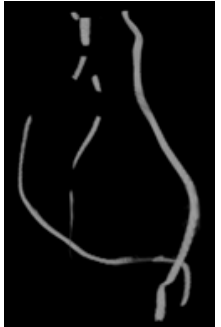

(e)

\begin{tabular}{|l|l|c|c|c|}
\hline Fig. & Description & Diameter & Abs. Error & Rel. Error \\
\hline (a) & gold standard (no motion) & $3.08 \mathrm{~mm}$ & - & - \\
(b) & periodic motion: classical method & $3.24 \mathrm{~mm}$ & $0.16 \mathrm{~mm}$ & $5.19 \%$ \\
(c) & periodic motion: proposed method & $3.24 \mathrm{~mm}$ & $0.16 \mathrm{~mm}$ & $5.19 \%$ \\
(d) & non-periodic motion: classical method & $1.85 \mathrm{~mm}$ & $1.23 \mathrm{~mm}$ & $39.94 \%$ \\
\hline (e) & non-periodic motion: proposed method & $3.42 \mathrm{~mm}$ & $0.34 \mathrm{~mm}$ & $11.04 \%$ \\
\hline
\end{tabular}

Fig. 1. Summary of the results of the phantom study. All volume renderings have common visualization settings. Details for the vessel diameter measurements and for the content of the images are listed in the table. The white arrow in the first image points to the measurement area of the vessel diameter.

\subsection{Results and Discussion}

Physical Heart Phantom. The volume rendering of the reconstruction results and the vessel diameter measurements are depicted in Fig. 1. For the case of periodic cardiac motion, both methods provide a similar reconstruction quality with a relative error of $5 \%$ in the vessel diameter. For the case of non-periodic motion, the proposed method clearly outperforms the periodic method. The relative error of the vessel diameter could be decreased by $29 \%$ from $40 \%(1.23 \mathrm{~mm})$ to $11 \%(0.34 \mathrm{~mm})$. This result is reasonable since our approach concerns the non-periodicity of the motion.

Clinical Cases: Arterial and Venous Vascular System. The volume rendering of the reconstruction results for the first case are shown in the upper row of Fig. 2, It can be seen that both motion compensated reconstruction methods are superior to the standard reconstruction without motion compensation. However, due to heart beat irregularities, our approach shows superior reconstruction results in terms of detail level and image artifacts.

The volume rendering of the reconstruction results for the second case are compared in the lower part of Fig. 2. It can be seen that the standard reconstruction shows blurring and artifical stenosises due to motion. The motion compensated reconstruction significantly reduces the motion artifacts and provides a higher level of detail. Due to the missing ECG signal no motion estimation and compensation could be performed with ECG-based perodic approaches. 


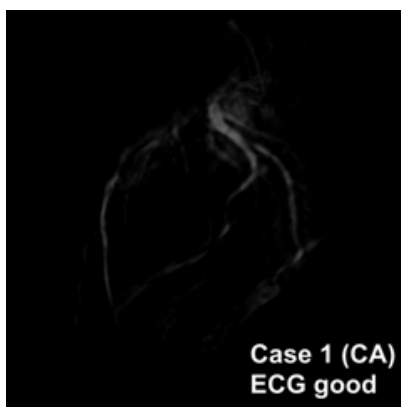

(a) standard reconstruction

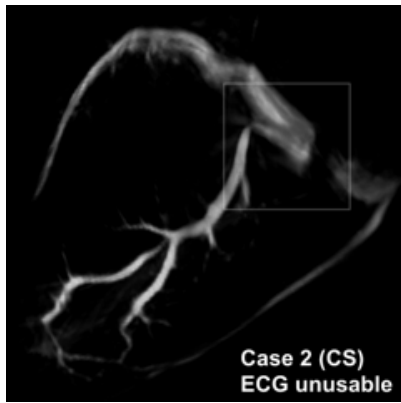

(d) standard reconstruction

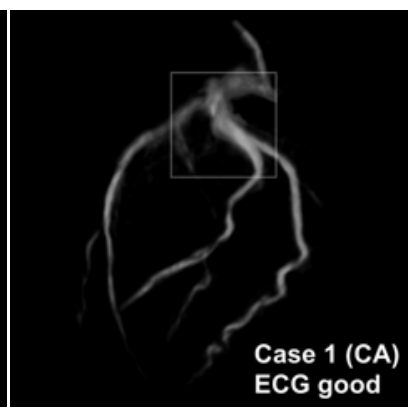

(b) classical estimation

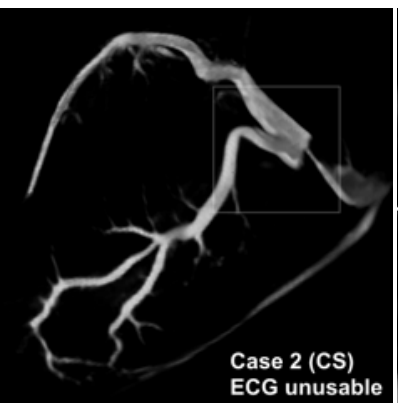

(e) proposed method

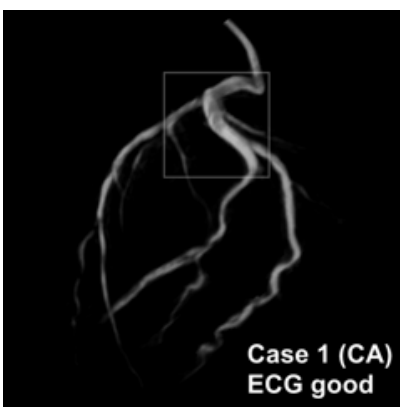

(c) proposed method

Fig. 2. Results for two clinical cases. The upper row depicts the volume rendering of a coronary artery (CA) dataset with a good ECG-signal using three different reconstruction methods. The images (d) (e) in the second row depict a coronary sinus (CS) case without ECG-signal. In (f) a zoomed-in version of the areas indicated by a square is depicted.

\section{Conclusion and Outlook}

The presented approach improves the reconstruction quality of dynamic high contrast objects significantly. This is achieved by two main contributions. First, the peridocity assumption was abandoned and replaced by a time-continous 4$\mathrm{D}$ motion model which is parameterized over the complete acquisition time. Second, a method which couples dynamic reconstruction and motion estimation was introduced. In our studies it proved to allow a robust estimation of the motion model parameters.

In a phantom and two clinical cases it could be shown that our method clearly improves the reconstruction quality in the presence of non-periodic motion and outperforms periodic approaches. In a phantom study the relative error of the vessel diameter could be decreased by $29 \%$ for non-cyclic motions.

The implications of our work for the development of future applications are diverse. Foremost, the reconstruction quality and stability in an interventional environment is increased. In long-term thinking, however, novel applications and 
diagnostic tools may be developed. A full 4-D model of the cardiac vasculature motion could be computed for deriving functional information. E.g. cardiac disfunctions could be detected by a 4-D animation over several heart beats. Further investigations will be subject of our upcoming research.

Disclaimer: The concepts and information presented in this paper are based on research and are not commercially available.

\section{References}

1. Strobel, N., Meissner, O., Boese, J., Brunner, T., Heigl, B., Hoheisel, M., Lauritsch, G., Nagel, M., Pfister, M., Rührnschopf, E.P., Scholz, B., Schreiber, B., Spahn, M., Zellerhoff, M., Klingenbeck-Regn, K.: 3D Imaging with Flat-Detector C-Arm Systems. In: Multislice CT, 3rd edn., pp. 33-51. Springer, Heidelberg (2009)

2. Desjardins, B., Kazerononi, E.: ECG-Gated Cardiac CT. American Journal of Roentgenology 182(4), 993-1010 (2004)

3. Schäfer, D., Borgert, J., Rasche, V., Grass, M.: Motion-compensated and gated cone beam filtered back-projection for 3-d rotational x-ray angiography. IEEE Transactions on Medical Imaging 25(7), 898-906 (2006)

4. Hansis, E., Schäfer, D., Grass, M., Dössel, O.: An iterative method for the reconstruction of the coronary arteries from rotational x-ray angiography. In: Hsieh, J., Flynn, M.J. (eds.) Proceedings of the SPIE Medical Imaging 2007: Physics of Medical Imaging, vol. 6510, p. 651026 (2007)

5. Blondel, C., Malandain, G., Vaillant, R., Ayache, N.: Reconstruction of coronary arteries from a single rotational x-ray projection sequence. IEEE Transactions on Medical Imaging 25(5), 653-663 (2006)

6. Prümmer, M., Wigstroem, L., Hornegger, J., Boese, J., Lauritsch, G., Strobel, N., Fahrig, R.: Cardiac C-arm CT: Efficient Motion Correction for 4D-FBP. In: Smith, G. (ed.) Nuclear Science Symposium, Medical Imaging, pp. 1-20 (2006)

7. Rohkohl, C., Lauritsch, G., Nöttling, A., Prümmer, M., Hornegger, J.: C-arm ct: Reconstruction of dynamic high contrast objects applied to the coronary sinus. In: IEEE NSS-MIC (Nuclear Science Symposium-Medical Imaging Conference), October 2008, p. M10-328 (2008)

8. Jandt, U., Schäfer, D., Grass, M., Rasche, V.: Automatic generation of time resolved $4 \mathrm{~d}$ motion vector fields of coronary arteries. In: 9th Int. Meeting on Fully 3D Image Reconstruction in Radiology and Nuclear Medicine, Lindau, Germany, pp. 249-252 (2007)

9. Hansis, E., Schäfer, D., Dössel, O., Grass, M.: Projection-based motion compensation for gated coronary artery reconstruction from rotational x-ray angiograms. Physics in Medicine and Biology 53(14), 3807-3820 (2008)

10. Unser, M.: Splines: A perfect fit for signal and image processing. IEEE Signal Processing Magazine 16(6), 22-38 (1999); IEEE Signal Processing Society's 2000 magazine award

11. Byrd, R.H., Lu, P., Nocedal, J., Zhu, C.: A limited memory algorithm for bound constrained optimization. SIAM J. Sci. Comput. 16(5), 1190-1208 (1995)

12. Scherl, H., Keck, B., Kowarschik, M., Hornegger, J.: Fast GPU-Based CT Reconstruction using the Common Unified Device Architecture (CUDA). In: Frey, E.C. (ed.) Nuclear Science Symposium, Medical Imaging Conference 2007, pp. 44644466 (2007) 